\title{
Evaluation of motor imagery ability in neurological patients: a review
}

\author{
Elke Heremans $^{1}$, Sarah Vercruysse ${ }^{1}$, Joke Spildooren ${ }^{1}$, Peter Feys ${ }^{2}$, Werner F Helsen ${ }^{3}$ and Alice Nieuwboer $^{1}$ \\ 1 Department of Rehabilitation Sciences, Katholieke Universiteit Leuven, Leuven, Belgium \\ 2 REVAL Rehabilitation \& Healthcare Research Center, PHL-University College, \& BIOMED, Universiteit Hasselt, Hasselt, \\ Belgium \\ 3 Department of Biomedical Kinesiology, Katholieke Universiteit Leuven, Leuven, Belgium
}

Received 5 February 2012 - Accepted 21 November 2012

\begin{abstract}
Motor imagery is a promising new intervention strategy within neurological rehabilitation. However, previous studies have shown that the ability to perform motor imagery is not well preserved in all neurological patients. Therefore, patients' motor imagery ability needs to be thoroughly examined when they are included in motor imagery rehabilitation programs or studies. In the past, objective methods to evaluate motor imagery were lacking rigour, and participants' imagery ability was often insufficiently assessed. The present paper discusses several methods to assess motor imagery ability and discusses their specific advantages and disadvantages. As well, an overview is given of studies applying these methods in patients with stroke, Parkinson's disease and multiple sclerosis.
\end{abstract}

Key words: Motor imagery, eye movements, hand movements, mental practice, imagery ability, neurorehabilitation

Résumé. Évaluation des capacités d'imagerie motrice chez les patients victimes d'une atteinte neurologique.

L'imagerie motrice est une technique prometteuse pour la réadaptation des patients victimes d'une atteinte neurologique. Plusieurs études ont cependant montré que la capacité à utiliser efficacement l'imagerie motrice n'est pas nécessairement préservée chez tous les patients. Aussi, il est indispensable de l'évaluer objectivement avant d'incorporer un travail mental au cours des programmes de rééducation. Par le passé, les méthodes objectives utilisées pour évaluer les capacités individuelles d'imagerie ont souvent manqué de rigueur. Parfois, ces capacités n'étaient même pas considérées. Cet article présente les avantages et inconvénients des différentes méthodes permettant de le faire. Les applications chez les patients ayant subi un accident vasculaire cérébral et les personnes atteintes de la maladie de Parkinson ou de la sclérose en plaques sont également abordées.

Mots clés : Imagerie motrice, mouvements oculaires, mouvements de la main, pratique mentale, capacité d'imagerie, neuro-réhabilitation

\section{Introduction}

Neurorehabilitation is based on the idea that cortical representations in the adult brain are not fixed but highly dynamic (Spitzer, 1999). Cortical connections and responses are being reorganized continuously as a result of peripheral and central alterations of input to the motor system (Munzert, Lorey, \& Zentgraf, 2009). In the past, a wide range of rehabilitation approaches have been used to provide precisely targeted input to the motor system as to optimise neuroplasticity in neurological patients (Robertson \& Murre, 1999). Recently, studies have shown that the motor system can even be activated when movements are not overtly executed, such as during motor imagery (MI). MI has been defined as a dynamic state during which a subject simulates an action mentally without any body movement (Jeannerod, 1994). According to the functional equivalence theory, motor imagery partly involves the same neural network that is used in actual perception and motor control, and can also activate neural circuits used in memory and emotion (Kosslyn, Ganis, \& Thompson, 2001; Moran, Guillot, Mac Intyre, \& Collet, 2012; Murphy, Nordin, \& Cumming, 2006). As a result, 
imagery can provide an alternative source of information that might be used for motor training aiming to improve performance and enhance neuroplasticity.

MI can be performed in different modalities and from either a first or third person visual perspective (Moran, et al., 2012). During kinesthetic MI, the individual imagines the feelings or sensations associated with a specific movement. In contrast, visual MI can be described as imagining seeing the performance of a task. Visual MI can be performed from a first or third person perspective, whereby the person imagines either being inside his/her body or views him/herself from the perspective of an external observer. As well, a distinction can be made between explicit MI, where the action is imagined consciously (e.g., voluntary active imagination of reaching for a cup), and implicit motor imagery, where MI is performed implicitly (e.g., answering a question about the handedness of a picture) (de Vries, Tepper, Otten, \& Mulder, 2011). Up to now, the effects of different imagery types and perspectives on the performance of different tasks are unclear (Moran, et al., 2012).

In athletes, different types of MI have been used for many years as an adjunct to other types of training (Murphy et al., 2006; Weinberg, 2008). Athletes can use MI either to prepare for an immediately upcoming action, since it primes the neural circuitry that needs to be activated during actual execution of the task, or for the actual training of motor skills. Motor imagery can be used throughout the different stages of learning. It might be used to acquire new skills as well as to assist a person to consolidate known strategies or to correct errors. A meta-analysis by Driskell, Copper, and Moran (1994) provided evidence on the positive effects of MI practice on the athletes' performance. Specifically, MI practice was shown to improve motor aspects such as strength, timing, accuracy and efficiency (Gentili, Han, Schweighofer, \& Papaxanthis, 2010; Yagüez, et al., 1998; Yue \& Cole, 1992), as well as motivational aspects (Rogers, 2006). The effectiveness of MI was shown to be moderated by several aspects, e.g. the type and complexity of the task, task familiarity, retention interval between practice and performance, the length and duration of the mental practice intervention and the imagery perspective (Driskell, et al., 1994; Olsson \& Nyberg, 2010; Weinberg, 2008).

\section{Motor imagery as a new therapy tool within neurological rehabilitation?}

In the last decade, also in the field of neurological rehabilitation, the interest in MI practice has greatly increased (e.g., Braun, Beurskens, Borm, Schack, \& Wade, 2006; Dickstein \& Deutsch, 2007; Jackson, Lafleur, Malouin, Richards, \& Doyon, 2001; Simmons, Sharma, Baron, \& Pomeroy, 2008). Up to now, most evidence was found regarding its use for patients recovering from a stroke. In stroke rehabilitation, MI has provided promising results for the training of a variety of tasks. Dunsky, Dickstein, Ariav, Deutsch, and Marcovitz (2006) reported that MI practice might be used to improve gait performance in individuals with post-stroke hemiparesis. After six weeks of MI practice, the patients showed an increase in walking speed, stride length, cadence and single support time on the affected lower limb, while decreasing their doublesupport time. A randomised clinical trial by Liu, Chan, Lee, and Hui-Chan (2004) showed that MI can be used after a stroke as a training strategy to promote the relearning of activities of daily living, such as washing the dishes or folding the laundry. In addition, in a review of Braun, et al. (2006), an overview was given on the evidence of MI practice as an additional therapy intervention to improve the recovery of arm function after stroke. However, caution is warranted since most of the abovementioned findings were based on research on small subgroups of patients, and a recent carefully designed randomised controlled trial by Ietswaart, et al. (2011) did not confirm these initial positive results. As such, there definitely remains a need for additional large-scale randomised controlled trials in this field. Furthermore, previous research has shown that imagery could not be universally applied to all stroke patients. For example, MI ability was shown to be impaired in patients with contralateral parietal and left prefrontal lesions (Johnson, 2000; Sirigu, et al., 1996). In line with these findings, Simmons, et al. (2008) reported that in $40 \%$ of the stroke patients the accuracy or temporal coupling of the imagined movements was disturbed as a consequence of the stroke. This phenomenon was labeled "chaotic motor imagery".

Another neurological population for which the use of MI has been investigated are patients with Parkinson's disease (PD). In a study of Tamir, Dickstein, and Huberman (2007), promising results were found regarding the use of MI practice in PD rehabilitation. These authors showed that the combination of MI and real practice can be effective in the treatment of PD, especially for reducing the patients' bradykinesia. Other studies, however, question the effectiveness of MI practice for this group, as they showed impairments in MI ability in patients with PD (Frak, Cohen, \& Pourcher, 2004). Dysfunction of the basal ganglia, which is the core deficit underlying $\mathrm{PD}$, might affect imagery performance, since several studies have shown that these nuclei are one of the central elements of a sensorimotor network that is involved in MI and widely overlaps with that involved in physical execution of the same movements (Gerardin, et al., 2000; Grèzes \& Decety, 2001; Kühn, et al., 2006; Leiguarda, Cerquetti, Tenca, \& Merello, 2009; Li, 2000). Decety (1996) showed that the basal ganglia are linked to the internal selection of sequential motor programs during MI. As such, it is not unlikely that the nigrostriatal dopaminergic deficiency of PD may affect imagery performance. Up to now, however, studies on this topic are scarce and have reported largely inconsistent results (Heremans, et al., 2011; Tamir, et al., 2007; Yagüez, Canavan, Lange, \& Hömberg, 1999). 
A third neurological population for which MI practice might be of use are patients with multiple sclerosis (MS). However, so far, hardly any studies have been performed examining MI in this group. A potential problem with regard to the use of MI in MS, is the high occurrence of cognitive problems in patients suffering from this disease. Cognitive impairments are present in $40-70 \%$ of the MS patients and mainly relate to problems in information processing speed, learning, memory and executive functioning (Calabrese, 2006). Heremans, D'hooge, De Bondt, Helsen, and Feys (2012) reported a significant correlation between MI accuracy and impairments in cognitive functioning in patients with MS. As such, it can not be taken for granted that MI could be successfully used in all patients with MS.

Overall, in all three of the abovementioned clinical groups, there remains uncertainty about the effects of MI interventions as well as on the effects of the neurological pathology on the patients' ability to use MI practice. This is partly due to the fact that many studies did not assess patients' imagery ability in a structured and objective way. If patients with diminished MI ability are included in an intervention study, the effects of the MI intervention on a group level are masked. As well, many studies did not perform any manipulation checks on imagery compliance as to validate their experimental instructions. To overcome these limitations, in future studies examining the use of MI in neurorehabilitation, the MI ability of the participants should be assessed in detail. In the next part, an overview will be given on the different methods to assess MI ability, and the advantages and disadvantages of each method will be discussed.

\section{Evaluation of motor imagery ability}

As the capacity to elicit efficient mental images differs widely in neurological patients, it is important to utilize appropriate psychological, behavioural and neurophysiological means to evaluate the participants' capacity in forming accurate mental images when using MI in training or research (Collet, Guillot, Lebon, MacIntyre, \& Moran, 2011; Guillot \& Collet, 2005a). In the past, most studies did not evaluate MI ability at all (e.g., Liu, et al., 2004; Tamir, et al., 2007), or evaluated it by means of subjective methods such as questionnaires and interviews (e.g., Page, Levine, Sisto, \& Johnston, 2001). An exception to this are studies that used the recording of autonomic responses to evaluate MI (see Collet, et al., 2011 and Guillot \& Collet, 2005a for reviews). Furthermore, an alternative method to objectively assess MI ability in a lab setting was developed by Heremans, Helsen, and Feys (2008), using eye-movement registration to assess MI.

Secondly, the studies that did take into account the participants' MI ability, most often evaluated only one aspect of imagery such as imagery vividness. However, MI has a multifaceted nature, which requires a combination of several methods to analyse this mental process in detail. Therefore, in a follow-up study, Heremans et al. (2011) also developed a comprehensive test battery to assess the different aspects of MI ability. In the following part, we will first discuss the newly developed and objective method to assess MI based on eye-movement registration. This method is mainly suitable to be used in a research context. Next, we will discuss the comprehensive MI ability test battery which is composed of different tasks and can be implemented easily in clinical practice. We will discuss the results of studies applying these methods in healthy persons as well as in two clinical groups, i.e. patients with $\mathrm{PD}$ and patients with MS.

\subsection{Eye-movement registration to assess MI ability in neurological patients}

In most motor imagery research, a precise and objective monitoring tool of the compliance during and ability of subjects to perform MI is lacking. Therefore, in a previous study, Heremans, et al. (2008) performed an alternative approach to objectively monitor the MI process online, based on the technique of eye-movement registration. This approach was based on two lines of evidence. First of all, previous research on movement execution has shown a very tight coupling, both spatially and temporally, between eye and hand movements during goal-directed upper limb actions, suggesting a common underlying command structure (Flanagan \& Johansson, 1999; Helsen, Elliott, Starkes, \& Ricker, 2000; Helsen, Feys, Heremans, \& Lavrysen, 2010). When developing our method based on eye-movement registration we hypothesized that this coupling would remain intact when goaldirected movements would be merely imagined instead of physically executed. Second, previous studies showed that eye-movement data can provide an excellent on-line indication of other cognitive processes, such as those underlying visual search and reading (Liversedge \& Findlay, 2000). As well, eye-movement recording has been shown to provide useful information about other types of nonmotor imagery. It has, for example, been shown that eye movements reflect the content of the imagined stimulus during other types of imagery such as imagery of auditory scenes, recently viewed pictures and moving stimuli (De'Sperati, 2003; Laeng \& Teodorescu, 2002; Spivey \& Geng, 2001). These studies all confirmed that, during visual imagery of a stationary or dynamic scene, eye movements can be a precise marker of the spatiotemporal evolution of the underlying mental process. However, despite this evidence in related fields, it was not clear yet to what extent eye movements could provide a reflection of the content of people's MI. To investigate this, a group of healthy participants was instructed to either execute or imagine cyclical flexion-extension movements of the wrist. During this experiment, participants were seated in front of a computer screen, with their right arm fixated in an arm orthosis and moved their wrist between two targets, projected at the screen at 
a rhythm of $1 \mathrm{~Hz}$. They performed this task for three different inter-target distances and under four different conditions: i) visual MI with eyes open, ii) visual MI with eyes closed, iii) physical execution and iv) rest. During all conditions their eye movements were recorded by means of an electro-oculography device (Porti 7, TMSinternational, Enschede, The Netherlands; sample frequency $=1024 \mathrm{~Hz}$ ). The main results of this study were that more than $80 \%$ of the participants made task-related eye movements during MI, both when they kept their eyes open and closed (89 and 85\%, respectively). Furthermore, their eye movements during imagery showed remarkable similarities in eye-movement characteristics with those made during physical execution of the same task and differed significantly from the irregular eye movements that were made during rest. The number of eye movements during imagery did not differ from the number of eye movements during execution. As well, in the same way as during execution, during imagery the eye-movement amplitude and duration adapted nicely to the distance that had to be covered in the mind. Based on these results, it could be concluded that, for most healthy subjects, eye-movement registration can serve as an objective and real-time indicator of what they are actually doing during imagery. As such, it can be considered an objective technique to monitor MI.

Next, this new method was applied to investigate MI ability in two groups of neurological patients, i.e. patients with PD and patients with MS (Heremans, Nieuwboer, Feys, et al., 2012; Heremans, Nieuwboer, Spildooren, et al., 2012). Both groups physically executed and visually imagined the same task as described above, requiring cyclical goal-directed flexion-extension movements of the wrist. In both studies it was found that eyemovement registration nicely captured the patients' MI performance. In patients with $\mathrm{PD}$, their slowness in executing as well as imagining this task was reflected in higher eye-movement times. As well, both the temporal and spatial eye-movement parameters captured the differences in performance when the task needed to be imagined for different amplitudes and under different conditions, such as in the presence and absence of additional visual information regarding the task. In addition, in this study (Heremans, Nieuwboer, Feys, et al., 2012), similar results were found when using the eye-movement registration method during MI of another, more functional task, i.e. the Box and Block Task. During this task, participants have to pick up and transport blocks from one side of a box to another. Although the use of eye-movement registration definitely needs to be validated for others tasks as well, the fact that good results were also found for this more clinical task is promising with regard to the implementation of this method in other situations.

In a next study (Heremans, Nieuwboer, Spildooren, et al., 2012), eye-movement registration was used to assess MI quality in patients with MS. Also in this group, the eye-movement data were shown to be useful to capture differences in MI performance between the patients and controls. More specifically, the eye movements showed that patients with MS were slower and had a tendency to spatially overshoot imagined movements, perhaps reflecting an ataxic behaviour. When additional visual information was provided to cue the patients' movement amplitude, however, they were able to correct for their spatial and temporal inaccuracy. As such, the abovementioned two studies show how eye-movement registration can be used to assess MI ability in neurological patients, as well as to assess the effects of strategies to improve the quality of MI training.

\subsection{A clinical battery to assess motor imagery ability in neurological patients}

As described above, the method to evaluate MI based on eye-movement registration has been shown useful to assess MI in healthy persons as well as neurological patients. A disadvantage of this technique, however, is that its use remains limited to lab settings in which specialised equipment is available, and that the transfer to clinical practice is limited. Therefore, in another study (Heremans, et al., 2011), a test battery was composed which could be used to assess MI in neurological patients also in a clinical context. Furthermore, this test battery aimed at evaluating the various aspects of MI, such as accuracy, vividness and temporal organisation, as well as several types of MI, including visual and kinesthetic MI. It consisted of three components: i) the Kinesthetic and Visual Imagery Questionnaire (KVIQ) (Malouin, et al., 2007), ii) a Hand Rotation Test, and iii) a Mental Chronometry Task. This test battery was used to evaluate motor imagery in patients with PD and MS.

\subsubsection{Components of the motor imagery test battery}

\section{Component 1 - KVIQ}

The KVIQ (Malouin, et al., 2007) is a questionnaire in which imagery vividness is assessed by means of a 5 -point visual analogue scale, in which a score of 1 indicates a very clear image or very clear kinesthetic sensations and a score of 5 that the participant does not experience any image or sensation at all. Imagery vividness refers to the clarity, brightness, or intensity of the mental representation (Marks, 1973). The KVIQ assesses both visual and kinesthetic MI, as the questionnaire comprises 10 visual and 10 kinesthetic items. After a demonstration by the experimenter, the participant is always instructed to first physically execute the movement, then imagine it, and finally score the imagery vividness on the 5 -point scale. Lateralized items are performed at both body sides. As all movements can be performed in sitting position, the KVIQ is well suited to assess imagery ability in populations with restricted mobility, among which part of the patients with neurological disorders. 
The main advantage of imagery questionnaires such as the KVIQ is that they are functional, easy and rapid to complete and give important information about the individual's personal experience with regard to MI vividness. The main disadvantage is that there remains an important part of subjectivity as participants perform an auto-evaluation of their MI vividness. Self-reports imply that individuals may overestimate their competence, or that answers may correlate with cognitive functioning or depression.

\section{Component 2 - Hand Rotation Test}

In this test, subjects are presented with 96 successive line drawings of hands (48 left and 48 right hands) in four different views (back, palm, ulnar, radial) and 12 different rotations (30 degree steps) (Nico, Daprati, Rigal, Parsons, \& Sirigu, 2004; Sharma, Jones, Carpenter, \& Baron, 2008). In our studies, the drawings were presented on a computer screen, as this allowed to both measure the accuracy and response time by means of E-prime 2.0 software and a serial response box (Psychology Software Tools), connected with a microphone. However, in a clinical setting, the pictures could also be presented on cards, so that the test can be performed at the patients' bedside. During the test, the participants are instructed to judge as fast and accurately as possible whether it concerns a picture of a left or right hand, and this without moving or seeing their own hands which rest on their laps with the palms down.

This mental rotation task has the advantage that it offers useful and objective information, while still being very easy to administer. Disadvantages, however, are that the test is highly subject to problems with left-right dissociation and that the strategies that people use to resolve the task are often unclear. Some patients might make use of alternative strategies that bypass the need for MI (Sharma, Pomeroy, \& Baron, 2006).

\section{Component 3 - Mental Chronometry Test}

As third part of the assessment, the participants performed an adapted version of the Box and Block Test (BBT) (Mathiowetz, Volland, Kashman, \& Weber, 1985), in which a mental chronometry paradigm was used. This technique is based on the temporal similarities between MI and actual execution of tasks. In the literature, this similarity is often referred to as "temporal isochrony". Mental chronometry is a useful and widely applied method to assess the timing preservation during MI (Decety, Jeannerod, \& Prablanc, 1989; Papaxanthis, Pozzo, Skoura, \& Schieppati, 2002). The BBT is performed using wooden blocks of $2.5 \mathrm{~cm}^{2}$ and a wooden box, which is divided in two equal parts by means of a partition with a height of $18 \mathrm{~cm}$. The box is placed at the participant's midline, with the compartment holding the blocks oriented towards the hand being tested. Unlike the original test where the number of transported blocks was counted within a time limit, the outcome of this test battery was the time needed to transport 20 blocks.
This method has the advantage that the degree of similarity between the duration of physically executing and visually imagining a task offers very objective information about the temporal organisation of the imagined performance. However, we should be aware that several factors may lead to an over- or underestimation of a movement's duration during MI (see Guillot \& Collet, 2005b for a review). Also, the fact that no clear cut off point has been defined to determine whether a difference between imagery and execution duration should be considered as abnormal, hampers the use of this technique in clinical practice. In addition, the information offered by this method is limited to the global movement instead of offering a detailed monitoring of the ongoing mental process. A final limitation is that mental chronometry does not provide any information about MI accuracy and vividness.

Importantly, when using mental chronometry, we should be aware that most evidence on its potential as a tool to quantify motor imagery was found in healthy persons (Decety, et al., 1989; Papaxanthis, et al., 2002). Studies in stroke patients showed mixed results. Malouin, Richards, Durand, and Doyon (2008) reported that the temporal congruence between imagined and executed tasks remained intact in stroke patients performing a relatively easy stepping task. Wu, Hermann, Ying, and Page (2010), on the other hand, found that using chronometry to monitor engagement in mental practice was not reliable in stroke patients performing more difficult tasks. So although the temporal congruence between actual and imagined times has been shown to provide a powerful and reliable assessment of imagery accuracy (Guillot \& Collet, 2005a, 2005b), implementation of this technique in neurological patients requires caution.

\subsubsection{Application of the motor imagery test battery in patients with $\mathrm{PD}$ and MS}

In previous work, this clinical test battery was already successfully used to assess MI ability in patients with PD and MS. In a first study (Heremans, et al., 2011), the MI ability of $14 \mathrm{PD}$ patients, in early and middle stages of the disease, was evaluated in comparison to 14 age-matched controls. All patients were in Hoehn and Yahr stages 1-3 and did not show any severe cognitive decline (Mini Mental State Examination score > 24) (Dick, et al., 1984). All tests were performed while the patients were in the onphase of their medication cycle. As main results, we found that the patients did not differ from the controls with respect to imagery vividness (measured by the KVIQ) and imagery accuracy (measured by the Hand Rotation Test). The BBT however revealed a significant temporal difference between both groups. PD patients were markedly slower when imagining movements than controls, in line with a slower execution.

In a next study (Heremans, D'hooge et al., 2012), the test battery was used in a group of 30 MS patients 
and 30 age-matched controls. Again, the battery revealed significant differences in imagery performance between the groups. Although no differences were found in imagery vividness by means of the KVIQ, MS patients were shown to have significantly lower imagery accuracy (measured by the Hand Rotation Test) than controls. Patients' lack of imagery accuracy correlated with cognitive disturbances. Furthermore, the BBT revealed differences in the temporal organization of MI, as patients were shown to be significantly slower during MI than controls and showed differences between their most and least affected body sides, indicating an association between their temporal parameters of MI and their motor functioning.

\section{Conclusion}

Previous studies (Dickstein \& Deutsch, 2007; Mulder, 2007), showed promising results with respect to the use of $\mathrm{MI}$ as a complement in physical rehabilitation programs. However, several studies (Johnson, 2000; Simmons, et al., 2008; Sirigu, et al., 1996) also revealed that MI ability is not well preserved in all neurological patients. As such, a thorough screening of patients' MI ability is recommended to identify those patients who might not benefit from this technique, or who need additional training with this method first before using it as a training tool. As well, a thorough screening of participants' MI ability is needed when performing research on the effect of MI programs, as a large variety in MI ability within the investigated sample might confound the results of these studies.

In the current paper we presented various methods to evaluate and monitor MI and discussed the advantages and disadvantages of each approach. Both the eyemovement registration method and the composite test battery were shown to be useful to assess MI in clinical groups, i.e. patients with PD and MS. The method based on eye-movement registration can mainly be recommended when assessing participants' MI ability in a research context. The composite test battery, on the other hand, is aimed to be an instrument to evaluate MI ability in neurological patients in a clinical setting.

\section{Bibliography}

Braun, S.M., Beurskens, A.J., Borm, P.J., Schack, T., \& Wade, D.T. (2006). The effects of mental practice in stroke rehabilitation: a systematic review. Archives of Physical Medicine and Rehabilitation, 87, 842-852.

Calabrese, P. (2006). Neuropsychology of multiple sclerosis An overview. Journal of Neurology, 253 (Suppl 1), I10-I15.

Collet, C., Guillot, A., Lebon, F., MacIntyre, T., \& Moran, A. (2011). Measuring motor imagery using psychometric, behavioral, and psychophysiological tools. Exercise and Sport Sciences Reviews, 39, 85-92.

Decety, J. (1996). The neurophysiological basis of motor imagery. Behavioural Brain Research, 77, 45-52.
Decety, J., Jeannerod, M., \& Prablanc, C. (1989). The timing of mentally represented actions. Behavioural Brain Research, 34, 35-42.

De'Sperati, C. (2003). Precise oculomotor correlates of visuospatial mental rotation and circular motion imagery. Journal of Cognitive Neuroscience, 15, 1244-1259.

De Vries, S., Tepper, M., Otten, B., \& Mulder, T. (2011). Recovery of motor imagery ability in stroke patients. Rehabilitation Research and Practice, 283840.

Dick, T.P.R., Guiloff, R.J., Stewart, A., Blackstock, J., Bielawska, C., Paul, E.A., \& Marsden, C.D. (1984). Mini-Mental State Examination in neurological patients. Journal of Neurology, Neurosurgery and Psychiatry, 47, 496-499.

Dickstein, R., \& Deutsch, J.E. (2007). Motor imagery in physical therapist practice. Physical Therapy, 87, 942-953.

Driskell, J.E., Copper, C., \& Moran, A. (1994). Does mental practice enhance performance? The Journal of Applied Psychology, 79, 481-492.

Dunsky, A., Dickstein, R., Ariav, C., Deutsch, J., \& Marcovitz, E. (2006). Motor imagery practice in gait rehabilitation of chronic post-stroke hemiparesis: four case studies. International Journal of Rehabilitation Research, 29, $351-356$.

Flanagan, J.R., \& Johansson, R.S. (1999). Gaze-hand coordination subserving motion planning in object manipulation. Society for Neuroscience Abstract, 29, 50.18.

Frak, V., Cohen, H., \& Pourcher, E. (2004). A dissociation between real and simulated movements in Parkinson's disease. Neuroreport, 15, 1489-1492.

Gentili, R., Han, C.E., Schweighofer, N., \& Papaxanthis, C. (2010). Motor learning without doing: trial-by-trial improvement in motor performance during mental training. Journal of Neurophysiology, 104, 774-783.

Gerardin, E., Sirigu, A., Lehéricy, S., Poline, J.B., Gaymard, B., Marsault, C., Agid, Y., \& Le Bihan, D. (2000). Partially overlapping neural networks for real and imagined hand movements. Cerebral Cortex, 10, 1093-1104.

Grèzes, J., \& Decety, J. (2001). Functional anatomy of execution, mental simulation, observation, and verb generation of actions: a meta-analysis. Human Brain Mapping, 12, $1-19$.

Guillot, A., \& Collet, C. (2005a). Contribution from neurophysiological and psychological methods to the study of motor imagery. Brain Research Reviews, 50, 387-397.

Guillot, A., \& Collet, C. (2005b). Duration of mentally simulated movement: a review. Journal of Motor Behavior, 37, $10-19$.

Helsen, W., Feys, P., Heremans, E., \& Lavrysen, A. (2010). Eye-hand coordination in goal-directed action: normal and pathological functioning. In D. Elliott \& M. Khan (Eds.), Vision and goal-directed movement: neurobehavioral perspectives (pp. 193-217). Champaign: Human Kinetics.

Helsen, W.F., Elliott, D., Starkes, J.L., \& Ricker, K.L. (2000). Coupling of eye, finger, elbow and shoulder movements 
during manual aiming. Journal of Motor Behavior, 32, $241-248$.

Heremans, E., D'hooge, A.M., De Bondt, S., Helsen, W.F., \& Feys, P. (2012). The relation between cognitive and motor dysfunction and motor imagery ability in patients with multiple sclerosis. Multiple Sclerosis Journal, 18, 1303-1309.

Heremans, E., Feys, P., Nieuwboer, A., Vercruysse, S., Vandenberghe, W., Sharma, N., \& Helsen, W.F. (2011). Motor imagery ability in patients with early and mid stage Parkinson's disease. Neurorehabilitation and Neural Repair, 25(2), 168-177.

Heremans, E., Helsen, W.F., \& Feys, P. (2008). The eyes as a mirror of our thoughts: quantification of motor imagery of goal-directed movements through eye movement registration. Behavioural Brain Research, 18\%, 351-360.

Heremans, E., Nieuwboer, A., Feys, P., Vercruysse, S., Vandenberghe, W., Sharma, N., \& Helsen, W.F. (2012). External cueing improves motor imagery quality in patients with Parkinson's disease. Neurorehabilitation and Neural Repair, 26(1), 27-35.

Heremans, E., Nieuwboer, A., Spildooren, J., De Bondt, S., D'hooge, A.M., Helsen, W.F., \& Feys, P. (2012). Cued motor imagery in patients with multiple sclerosis. Neuroscience, 206, 115-121.

Ietswaart, M., Johnson, M., Dijkerman, H.C., Joice, S., Scott, C., MacWalter, S., \& Hamilton, S. (2011). Mental practice with motor imagery in stroke recovery: randomised controlled trial of efficacy. Brain, 134, 1373-1386.

Jackson, P.L., Lafleur, M.F., Malouin, F., Richards, C., \& Doyon, J. (2001). Potential role of mental practice using motor imagery in neurologic rehabilitation. Archives of Physical Medicine and Rehabilitation, 82, 1133-1141.

Jeannerod, M. (1994). The representing brain: Neural correlates of motor intention and imagery. Behavioural and Brain Sciences, 17, 187-245.

Johnson, S.H. (2000). Imagining the impossible: intact motor representations in hemiplegics. Neuroreport, 11, 729-732.

Kosslyn, S.M., Ganis, G., \& Thompson, W.T. (2001). Neural foundations of imagery. Nature Reviews. Neuroscience, 2, $635-642$.

Kühn, A., Doyle, L., Pogosyan, A., Yarrow, K., Kupsch, A., Schneider, G.-H., Hariz, M.I., Trottenberg, T., \& Brown, P. (2006). Modulation of beta oscillations in the subthalamic area during motor imagery in Parkinson's disease. Brain, 129, 695-706.

Laeng, B., \& Teodorescu, D.S. (2002). Eye scanpaths during visual imagery reenact those of perception of the same visual scene. Cognitive Sciences, 26, 207-231.

Leiguarda, R., Cerquetti, D., Tenca, E., \& Merello, M. (2009). Globus pallidus internus firing rate modification after motor-imagination in three Parkinson's disease patients. Journal of Neural Transmission, 116, 451-455.

Li, C.R. (2000). Impairment of motor imagery in putamen lesions in humans. Neuroscience Letters, 28\%, 13-16.
Liu, K.P., Chan, C.C., Lee, T.M., \& Hui-Chan, C.W. (2004). Mental imagery for promoting relearning for people after stroke: a randomized controlled trial. Archives of Physical Medicine and Rehabilitation, 85, 1403-1408.

Liversedge, S.P., \& Findlay, J.M. (2000). Saccadic eye movements and cognition. Trends in Cognitive Sciences, 4, $6-14$.

Malouin, F., Richards, C.L., Durand, A., \& Doyon, J. (2008). Reliability of mental chronometry for assessing motor imagery ability after stroke. Archives of Physical Medicine and Rehabilitation, 89, 311-319.

Malouin, F., Richards, C.L., Jackson, P.L., Lafleur, M., Durand, A., \& Doyon, J. (2007). The Kinesthetic and Visual Imagery Questionnaire (KVIQ) for assessing motor imagery in persons with physical disabilities: a reliability and construct validity study. Journal of Neurologic Physical Therapy, 31, 20-29.

Marks, D.F. (1973). Visual imagery differences in the recall of pictures. British Journal of Psychology, 64, 17-24.

Mathiowetz, V., Volland, G., Kashman, N., \& Weber, K. (1985). Adult norms for the Box and Block Test of manual dexterity. The American Journal of Occupational Therapy, 39, 386-391.

Moran, A., Guillot, A., Mac Intyre, T., \& Collet, C. (2012). Re-imagining mental imagery: building bridges between cognitive neuroscience and sport psychology. British Journal of Psychology, 103, 224-247.

Mulder, T. (2007). Motor imagery and action observation: cognitive tools for rehabilitation. Journal of Neural Transmission, 114, 1265-1278.

Munzert, J., Lorey, B., \& Zentgraf, K. (2009). Cognitive motor processes: the role of motor imagery in the study of motor representations. Brain Research Reviews, 60, 306-326.

Murphy, S.M., Nordin, S.M., \& Cumming, J. (2006). Imagery in sport, exercise and dance. In T. Horn (Ed.), Advances in sport psychology (3rd ed.). Champaign, IL: Human Kinetics.

Nico, D., Daprati, E., Rigal, F., Parsons, L., \& Sirigu, A. (2004). Left and right hand recognition in upper limb amputees. Brain, 127, 120-132.

Olsson, C.J., \& Nyberg, L. (2010). Motor imagery: if you can't do it, you won't think it. Scandinavian Journal of Medicine and Science in Sports, 20, 711-715.

Page, S.J., Levine, P., Sisto, S.A., \& Johnston, V. (2001). A randomized efficacy and feasibility study of imagery in acute stroke. Clinical Rehabilitation, 15, 233-240.

Papaxanthis, C., Pozzo, T., Skoura, X., \& Schieppati, M. (2002). Does order and timing in performance of imagined and actual movements affect the motor imagery process? The duration of walking and writing tasks. Behavioural Brain Research, 134, 209-215.

Robertson, I.H., \& Murre, J.M. (1999). Rehabilitation of brain damage: brain plasticity and principles of guided recovery. Psychological Bulletin, 125, 544-575. 
Rogers, R.G. (2006). Mental practice and acquisition of motor skills: examples from sports training and surgical education. Obstetrics and Gynecology Clinics of North America, 33, 297-304.

Sharma, N., Jones, P.S., Carpenter, T.A., \& Baron, J.C. (2008). Mapping the involvement of BA $4 \mathrm{a}$ and $4 \mathrm{p}$ during motor imagery. Neuroimage, 41, 92-99.

Sharma, N., Pomeroy, V.M., Baron, J.C. (2006). Motor imagery: a backdoor to the motor system after stroke? Stroke, 37, 1941-1952.

Simmons, L., Sharma, N., Baron, J.C., \& Pomeroy, V.M. (2008). Motor imagery to enhance recovery after subcortical stroke: who might benefit, daily dose, and potential effects. Neurorehabilitation and Neural Repair, 22, 458-467.

Sirigu, A., Duhamel, J.R., Cohen, L., Pillon, B., Dubois, B., \& Agid, Y. (1996). The mental representation of hand movements after parietal cortex damage. Science, 273, 1564-1568.

Spitzer, M. (1999). The mind within the net: Models of learning, thinking, and acting. Cambridge, MA: MIT Press.

Spivey, M.J., \& Geng, J.J. (2001). Oculomotor mechanisms activated by imagery and memory: eye movements to absent objects. Psychological Research, 65, 235-241.
Tamir, R., Dickstein, R., \& Huberman, M. (2007). Integration of motor imagery and physical practice in group treatment applied to subjects with Parkinson's disease. Neurorehabilitation and Neural Repair, 21, 68-75.

Weinberg, R.S. (2008). Does imagery work? Effects on performance and mental skills. Journal of Imagery Research in Sport and Physical Activity, 3, 1-21.

Wu, A.J., Hermann, V., Ying, J., \& Page, S.J. (2010). Chronometry of mentally versus physically practiced tasks in people with stroke. The American Journal of Occupational Therapy, 64, 929-934.

Yagüez, L., Canavan, A.G.M., Lange, H.W., \& Hömberg, V. (1999). Motor learning by imagery is differentially affected in Parkinson's and Huntington's diseases. Behavioural Brain Research, 102, 115-127.

Yagüez, L., Nagel, D., Hoffman, H., Canavan, A.G.M., Wist, E., \& Hömberg, V. (1998). A mental route to motor learning: improving trajectorial kinematics through imagery training. Behavioural Brain Research, 90, 95-106.

Yue, G., \& Cole, K.J. (1992). Strength increases from the motor program: comparison of training with maximal voluntary and imagined muscle contractions. Journal of Neurophysiology, 67, 1114-1123. 